University of Nebraska - Lincoln

DigitalCommons@University of Nebraska - Lincoln

March 1997

\title{
Three-body dispersion coefficients for alkali-metal atoms
}

\author{
Mircea Marinescu \\ University of Nebraska - Lincoln \\ Anthony F. Starace \\ University of Nebraska-Lincoln, astarace1@unl.edu
}

Follow this and additional works at: https://digitalcommons.unl.edu/physicsstarace

Part of the Physics Commons

Marinescu, Mircea and Starace, Anthony F., "Three-body dispersion coefficients for alkali-metal atoms" (1997). Anthony F. Starace Publications. 58.

https://digitalcommons.unl.edu/physicsstarace/58

This Article is brought to you for free and open access by the Research Papers in Physics and Astronomy at DigitalCommons@University of Nebraska - Lincoln. It has been accepted for inclusion in Anthony F. Starace Publications by an authorized administrator of DigitalCommons@University of Nebraska - Lincoln. 


\title{
Three-body dispersion coefficients for alkali-metal atoms
}

\author{
Mircea Marinescu and Anthony F. Starace \\ Department of Physics and Astronomy, The University of Nebraska, 116 Brace Laboratory, Lincoln, Nebraska 68588-0111
}

(Received 10 July 1996)

\begin{abstract}
We study the nonadditive part of the long-range interaction among three alkali-metal atoms in their ground states. Using nondegenerate perturbation theory, up to the third order, we have computed the dispersion coefficients $C$ for three alkali-metal atoms interacting via their electric dipole moments. Both heteronuclear and homonuclear cases are considered. The numerical values for the $C$ coefficients suggest that such threebody dipole interaction effects may not be neglected in the description of the long-range surface potential interaction for alkali-metal atoms. Furthermore, we show that approximate formulas for $C$ of Midzuno and Kihara [J. Phys. Soc. Jpn. 11, 1045 (1956)] give results in excellent agreement with results of our calculations. Comparisons are given of our results with results of others and with results of other approximate formulas. The effect on our results for the $C$ coefficient of uncertainties in the experimental values of the static dipole polarizabilities, on which our results depend, is also analyzed. [S1050-2947(97)06003-4]

PACS number(s): 34.20.Cf, 31.15.-p, 33.90.+h
\end{abstract}

\section{INTRODUCTION}

Knowledge of the potential interaction among atoms plays an important role in the study of cold atom collision processes, which have applications to laser cooling and trapping of atoms. This paper is concerned with the study of the long-range interactions among three alkali-metal atoms in their ground states. The long-range limit of the potential surface interactions may be expressed in a power series in the inverses of the internuclear separations. The algebraic coefficient of each power combination in this series is a dispersion coefficient. These coefficients may be computed using perturbation theory, where the perturbation is given by the Coulomb interaction among the atomic charge distributions, and where the unperturbed Hamiltonian is given by the sum of the atomic Hamiltonians. (The unperturbed Hamiltonian becomes the exact one in the limit of infinite separation distances between the nuclei.) The perturbation parameters are proportional to the inverses of the internuclear distances. The dispersion series includes, in addition to the well-known pair-interaction coefficients $C_{6}, C_{8}$, and $C_{10}$ [1] (which result from second-order perturbation theory), a coefficient $C$ related to the electric dipole moment interactions among all three atoms. The $C$ coefficient results from third-order perturbation theory and describes the strength of the nonadditive, three-atom interaction.

The first investigations of the three-dipole interaction were by Axilrod and Teller [2] and by Muto [3] in 1943. Axilrod and Teller [2] give the order of magnitude of the constant $C$ and Muto [3] estimated its value using a simple atomic model. Axilrod [4] later also employed a simplified atomic model to derive the constant $C$, obtaining a value in agreement with that of Ref. [3]. Midzuno and Kihara [5] and Kihara [6] used a variational method to find an approximate expression for the coefficient $C$ in terms of the reduced masses and polarizabilities of the three interacting atoms. (We shall show in this paper that this approximate relationship is very accurate for the alkali-metal atom three-body systems.) Aub and Zienau [7] derived the three-body interaction energy for three neutral atoms by the methods of quantum electrodynamics. In the region in which the distances between atoms is small compared to the wavelengths corresponding to typical atomic excitations, their results agree with those obtained by Axilrod [4] (and hence also with those of Muto [3]). A compact expression for the $C$ coefficient (in terms of an integral over the product of the dynamic atomic dipole polarizabilities of imaginary frequencies) was established by McLachlan [8]. The first accurate evaluation of the $C$ coefficient was given by Chan and Dalgarno for three hydrogen atoms in 1965 [9] using a double integral representation for the $C$ coefficient. For further background material, we refer the interested reader to the review by Dalgarno and Davison [10]. We note here, however, that strong evidence for the significance of three-body interactions is provided by measurements of the third virial coefficient, particularly for low temperatures $[11,12]$.

For the alkali-metal atoms with which this paper is concerned, there have been a number of calculations since the late 1960s. All of these calculations aim to describe the dipole oscillator strength of a single alkali-metal atom as accurately as possible and then to use the results to compute the three-body dispersion coefficients. However, there have been only two ab initio calculations, both for Li: Stacey and Dalgarno [13] employed multiconfiguration wave functions and Yan et al. [14] employed Hylleraas wave functions. Also for Li, Margoliash et al. [15] employed a so-called "pseudospectral theory" to represent the then available dipole oscillator strength distribution data. For all of the alkali metals, Langhoff and Karplus [16] and Standard and Certain [17] have provided Padé approximant bounds on the three-body dispersion coefficients. These bounds, of course, depend on the experimental and theoretical input data used to generate the bounds. Finally, Tang [18] has analyzed the approximate Midzuno and Kihara [5,6] formula and presented two additional approximate formulas for the three-body dispersion coefficients. General discussions of such approximate formulas, known as "combination rules," have been presented by Kramer and Herschbach [19], by Margoliash et al. [15], and by Jhanwar and Meath [20]. Such approximate formulas are useful when $a b$ initio results are unavailable. 
In this paper we present the evaluation of the $C$ coefficients for all combinations of three interacting alkali-metal atoms in their ground states. The interactions of the valence electrons with the closed-shell cores of the alkali-metal atoms are described by $l$-dependent model potentials [1]. Section II presents the mathematical expression for the threedipole interaction coefficient $C$ as an integral over the product of atomic dipole polarizabilities of imaginary frequencies. The derivation of the $C$ coefficient expression presented here provides a complete picture of the contributions of the different orders of perturbation theory to the dispersion series of the long-range potential surface. Our final expression for the $C$ coefficient agrees with that of McLachlan [8]. Discussions about the evaluation of the $C$ coefficients are presented in Sec. III and values for the $C$ coefficients are given for all possible combinations of three alkali-metal atoms. Comparisons are also made to other results for alkalimetal atoms [13-17] as well as to results we have obtained using various combination rule formulas $[5,6,15,18-20]$. Section IV presents our conclusions.

Throughout this paper we use atomic units $\left(\hbar=m_{e}=c=1\right)$.

\section{THEORY}

In a Born-Oppenheimer picture the electronic Hamiltonian of the three interacting atoms is given by

$$
H=H_{1}+H_{2}+H_{3}+V_{12}+V_{23}+V_{31},
$$

where $H_{j}$, for $j=1,2,3$, is the Hamiltonian of the $j$ th atom and $V_{i j}$ is the Coulomb interaction between the atomic charge distributions of the $i$ th and $j$ th atoms. The eigenvalue of Eq. (1) as a function of the internuclear distances is the potential energy surface describing the interactions among the three atoms. We are concerned with the long-range behavior of the potential surface, for the case in which, in the dissociation limit, the atoms are in their ground state. Thus, in this limit, the eigenvalue problem may be solved using perturbation theory, where the unperturbed state is described by the sum of the atomic Hamiltonians, i.e., $\mathrm{H}_{1}+\mathrm{H}_{2}+\mathrm{H}_{3}$, and the perturbation by the sum of the Coulomb interactions between the atomic charge distributions. The perturbation parameters are proportional to the inverses of the internuclear distances.

The formalism and the final computation are made for a system of three alkali-metal atoms. The closed-shell cores of the alkali-metal atoms are described by $l$-dependent model potentials, which have been presented in Ref. [1]. Thus, only the valence electron will be taken explicitly into consideration. In the long-range limit, the Coulomb interaction between two hydrogenlike atoms may be written [21-23] as a powers series in $1 / R$,

$$
V\left(\vec{R}, \vec{r}_{1}, \vec{r}_{2}\right)=\sum_{l=1}^{\infty} \sum_{L=1}^{\infty} \frac{V_{l L}\left(\vec{r}_{1}, \vec{r}_{2}\right)}{R^{l+L+1}},
$$

where $R$ is the internuclear distance and where $\vec{r}_{1}$ and $\vec{r}_{2}$ are the position vectors of the electrons relative to their respective nuclei. $\vec{R}$ is assumed to be along the $z$ axis. In Eq. (2), the functions $V_{l L}$ are given by [24]

$$
\begin{aligned}
V_{l L}\left(\vec{r}_{1}, \vec{r}_{2}\right)=(-1)^{L} 4 \pi(\hat{l} \hat{L})^{-1 / 2} \\
\quad \times \sum_{m} K_{l L}^{m} r_{1}^{l} r_{2}^{L} Y_{l m}\left(\hat{r}_{1}\right) Y_{L-m}\left(\hat{r}_{2}\right),
\end{aligned}
$$

where

$$
K_{l L}^{m}=\left(C_{l+L}^{l+m} C_{l+L}^{L+m}\right)^{1 / 2}
$$

$\hat{l} \equiv 2 l+1, \quad$ and $\quad \hat{L} \equiv 2 L+1 . \quad$ The coefficients $\quad C_{n}^{k} \equiv$ $n ! / k !(n-k) !$ are the binomial coefficients.

The eigenfunctions of the unperturbed Hamiltonian (which is the sum of the atomic Hamiltonians) may be written as a product of the atomic wave functions, i.e.,

$$
\Psi_{\nu_{1} \nu_{2} \nu_{3}}^{(0)}=\Phi_{\nu_{1}}\left(\vec{r}_{1}\right) \Phi_{\nu_{2}}\left(\vec{r}_{2}\right) \Phi_{\nu_{3}}\left(\vec{r}_{3}\right)
$$

where $\nu$ is the triplet of atomic quantum numbers $\{n l m\}$ and $\Phi_{\nu}$ is the atomic wave function. The eigenvalue corresponding to the wave function in Eq. (5) is

$$
E_{\nu_{1} \nu_{2} \nu_{3}}^{(0)}=E_{\nu_{1}}+E_{\nu_{2}}+E_{\nu_{3}}
$$

where $E_{\nu}$ is the atomic energy corresponding to the atomic state $\nu$. Since we are interested in studying the long-range limit of the potential surface corresponding to the dissociation limit in which all three atoms are in their ground state, we have to consider the perturbation correction to the ground-state energy of the system, i.e., $\nu_{1}=\nu_{2}$ $=\nu_{3}=\nu_{g} \equiv\left\{n_{g} 00\right\}$. In this case the perturbation problem is not degenerate.

The first order correction to the energy is zero. The first nonzero contribution to the ground-state energy appears in

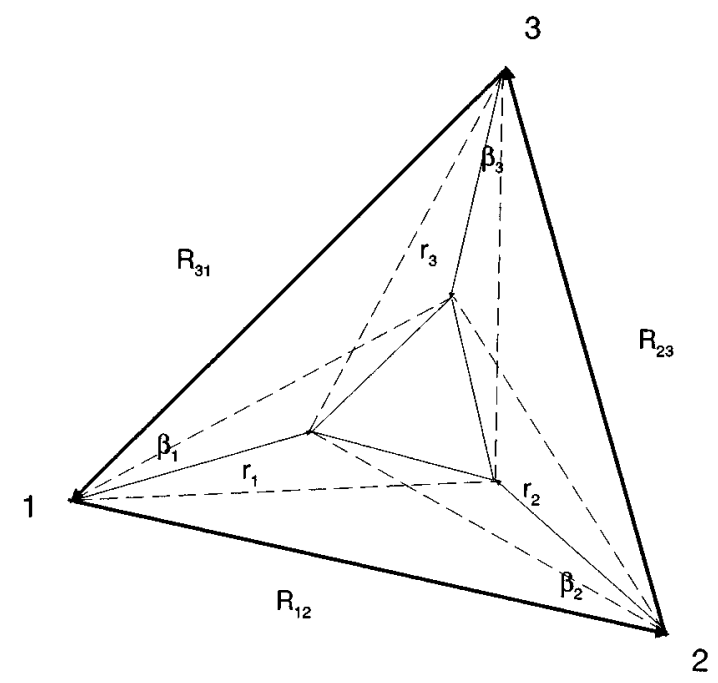

FIG. 1. Geometry of the three interacting alkali-metal atoms. The singly charged ions are located at 1,2 , and 3 and have internuclear separations $R_{i j}$. The angle $\beta_{j}$ is defined by $\cos \beta_{j} \equiv-\hat{R}_{i j} \cdot \hat{R}_{j k}$. The $i$ th valence electron is located at the position $\vec{r}_{i}$ relative to the $i$ th ion core. 
the second order of the perturbation. It has the following well-known expression in terms of the pair-interaction dispersion coefficients [1]:

$$
\begin{aligned}
E_{\nu_{g} \nu_{g} \nu_{g}}^{(2)}= & -\frac{C_{6}^{(12)}}{R_{12}^{6}}-\frac{C_{8}^{(12)}}{R_{12}^{8}}-\frac{C_{10}^{(12)}}{R_{12}^{10}}-\cdots-\frac{C_{6}^{(23)}}{R_{23}^{6}}-\frac{C_{8}^{(23)}}{R_{23}^{8}} \\
& -\frac{C_{10}^{(23)}}{R_{23}^{10}}-\cdots-\frac{C_{6}^{(31)}}{R_{31}^{6}}-\frac{C_{8}^{(31)}}{R_{31}^{8}}-\frac{C_{10}^{(31)}}{R_{31}^{10}}-\cdots,
\end{aligned}
$$

where $R_{12}, R_{23}$, and $R_{31}$ are the internuclear distances (see Fig. 1). The superscripts on the dispersion coefficients designate the atomic pair interaction to which they belong. For the case of three identical atoms the superscripts may be discarded. The $C_{6}$ coefficients describe the dipole-dipole in- teraction between two atoms, the $C_{8}$ coefficients the dipolequadrupole interaction, and the $C_{10}$ coefficients the sum of the dipole-octupole and quadrupole-quadrupole interactions.

Since the perturbation term in Eq. (1) is a sum of pairinteraction terms, the collective effect of all three atoms interacting appears in the third and higher orders of perturbation. We are concerned here with the computation of the lowest order, nonadditive contribution in inverse powers of the internuclear distances, which appears in the third order of perturbation. The third order correction to the ground-state energy may be written as

$$
E_{\nu_{g} \nu_{g} \nu_{g}}^{(3)}=\sum_{\sigma(123)} E_{\sigma}^{(3)}
$$

where $\sigma$ is a permutation of $(1,2,3)$ and the sum is taken over all possible permutations. In Eq. $(8), E_{\sigma}^{(3)}$ is given by

$$
E_{i j k}^{(3)}=\sum_{\nu_{i} \nu_{j} \nu_{k}} \frac{\left\langle\nu_{g} \nu_{g}\left|V^{(j k)}\right| \nu_{j} \nu_{k}\right\rangle\left\langle\nu_{g} \nu_{k}\left|V^{(k i)}\right| \nu_{i} \nu_{g}\right\rangle\left\langle\nu_{i} \nu_{j}\left|V^{(i j)}\right| \nu_{g} \nu_{g}\right\rangle}{\left(E_{\nu_{k}}+E_{\nu_{j}}-2 E_{\nu_{g}}\right)\left(E_{\nu_{i}}+E_{\nu_{j}}-2 E_{\nu_{g}}\right)}
$$

where $V^{(p q)}$ is the Coulomb interaction between the charge distributions of the $p$ th and $q$ th atoms, given by Eq. (2). Substituting Eq. (2) into Eq. (9), and keeping only the lowest term in inverse powers of the inter-nuclear distances, $E_{i j k}^{(3)}$ may be written as

$$
E_{i j k}^{(3)}=\frac{C_{i j k}}{R_{i j}^{3} R_{j k}^{3} R_{k i}^{3}},
$$

where

$$
\begin{aligned}
C_{i j k}= & \sum \frac{{ }^{(j k)} U_{n_{j} 1 m_{j} n_{k} 1 m_{k} ; 11}^{n_{g} 00 n_{g} 00} U_{n_{i} 1 m_{i} n_{g} 00 ; 11}^{n_{g} 00 n_{k} 1 m_{k}^{\prime}}{ }^{(i j)} U_{n_{g} 00 n_{g} 00 ; 11}^{n_{i} 1 m_{i}^{\prime} n_{j} 1 m_{j}^{\prime}}}{\left(E_{n_{k} 1}+E_{n_{j} 1}-2 E_{n_{g} 0}\right)\left(E_{n_{i} 1}+E_{n_{j} 1}-2 E_{n_{g} 0}\right)} \\
& \times d_{m_{k}^{\prime} m_{k}}^{1}\left(\pi-\beta_{k}\right) d_{m_{i}^{\prime} m_{i}}^{1}\left(\pi-\beta_{i}\right) d_{m_{j}^{\prime} m_{j}}^{1}\left(\beta_{j}-\pi\right),
\end{aligned}
$$

where we have used the $U$ symbols introduced in Appendix B of Ref. [24]. The sum from Eq. (11) is taken over $n_{i}, n_{j}, n_{k}, m_{i}, m_{i}^{\prime}, m_{j}, m_{j}^{\prime}, m_{k}$, and $m_{k}^{\prime}$. The superscript on the left side of each $U$ symbol designates the internuclear axis for which $U$ is computed. The rotation matrix $d_{m_{k}^{\prime} m_{k}}^{1}$ [25] comes from the fact that the $\Phi_{\nu_{k}}$ wave function appearing in the first matrix element in Eq. (9) is defined relative to the internuclear axis $(j k)$ as the $z$ axis while in the second matrix element in Eq. (9) it is defined relative to the $(k i)$ internuclear axis as the $z$ axis, the latter being rotated by the angle $\pi-\beta_{k}$ relative to the ( $\left.j k\right)$ axis (see Fig. 1). The rotation matrices with indices $i$ and $j$ have a similar origin. Using Eqs. (B10), (B14), and (B15) from Appendix B of Ref. [24], $C_{i j k}$ in Eq. (11) may be expressed as

$$
C_{i j k}=\mathcal{D}\left(\beta_{i}, \beta_{j}, \beta_{k}\right) S_{i j k},
$$

where

$$
\begin{aligned}
\mathcal{D}\left(\beta_{i}, \beta_{j}, \beta_{k}\right)= & -\frac{1}{27} \sum_{m_{i} m_{j} m_{i}^{\prime}} K_{11}^{m_{i}} K_{11}^{m_{j}} K_{11}^{m_{i}^{\prime}}(-1)^{m_{i}} \\
& \times d_{m_{i}-m_{j}}^{1}\left(\pi-\beta_{k}\right) d_{m_{i}^{\prime} m_{i}}^{1}\left(\pi-\beta_{i}\right) \\
& \times d_{-m_{i}^{\prime} m_{j}}^{1}\left(\beta_{j}-\pi\right)
\end{aligned}
$$

and

$$
S_{i j k}=\sum_{n_{i} n_{j} n_{k}} \frac{\left(n_{g} 0|r| n_{j} 1\right)^{2}\left(n_{g} 0|r| n_{k} 1\right)^{2}\left(n_{g} 0|r| n_{i} 1\right)^{2}}{\left(E_{n_{k} 1}+E_{n_{j} 1}-2 E_{n_{g} 0}\right)\left(E_{n_{i} 1}+E_{n_{j} 1}-2 E_{n_{g} 0}\right)} .
$$

In Eq. (14), the factors in the numerator are radial matrix elements. Using the analytic expressions for the $d^{1}$ functions [25] and Eq. (4) and making some elementary trigonometric transformations, we arrive at the following simple expression for $\mathcal{D}$ in Eq. (12):

$$
\mathcal{D}\left(\beta_{i}, \beta_{j}, \beta_{k}\right)=\frac{1}{9}\left(1+3 \cos \beta_{i} \cos \beta_{j} \cos \beta_{k}\right),
$$

which is symmetric under permutation of the angles. Thus, Eq. (8) becomes

$$
E_{\nu_{g} \nu_{g} \nu_{g}}^{(3)}=\frac{\mathcal{D}\left(\beta_{1} \beta_{2} \beta_{3}\right)}{R_{12}^{3} R_{23}^{3} R_{31}^{3}} \sum_{\sigma(123)} S_{\sigma},
$$

in which we have used the symmetry of the fraction to the left of the summation and specified that $i, j, k \equiv 1,2,3$. A convenient way to evaluate the sum in Eq. (14) is to separate it into independent contributions of each atom. This is not possible for each $S_{\sigma}$ individually but it is possible for the sum, 


$$
S=\sum_{\sigma(123)} S_{\sigma}
$$

by using the following integral representation: ${ }^{1}$

$$
\begin{gathered}
\frac{1}{(b+a)(c+a)}+\frac{1}{(a+b)(c+b)}+\frac{1}{(a+c)(b+c)} \\
=\frac{4}{\pi} \int_{0}^{\infty} d \omega \operatorname{Re}\left(\frac{1}{a-i \omega}\right) \operatorname{Re}\left(\frac{1}{b-i \omega}\right) \operatorname{Re}\left(\frac{1}{c-i \omega}\right),
\end{gathered}
$$

which applies for $a>0, b>0$, and $c>0$, and in which $\operatorname{Re}$ denotes the real part. By choosing $a=E_{n_{i} 1}-E_{n_{g} 0}$, $b=E_{n_{j} 1}-E_{n_{g} 0}$, and $c=E_{n_{k} 1}-E_{n_{g} 0}$ and applying Eq. (18) to the sum in Eq. (17), where each $S_{\sigma}$ is given by Eq. (14), inverting the summation and the integration, and computing formally the sums over the atomic principal quantum numbers, we finally get the following expression for $S$ :

$$
S=\frac{8}{\pi} \int_{0}^{\infty} d \omega\left\{\operatorname{Re}\left[\left(n_{g} 0\left|r g_{1}\left(E_{n_{g} 0}+i \omega\right) r\right| n_{g} 0\right)\right]\right\}^{3},
$$

where $g_{1}$ is a radial Green's function for angular momentum $l=1$ and for the complex energy $E_{n_{g} 0}+i \omega$. The radial matrix element on the right-hand side of Eq. (19) is proportional to the atomic dynamic dipole polarizability $\alpha_{1}$ for the ground state, evaluated at an imaginary frequency $i \omega$. Thus Eq. (19) may be written also as

$$
S=\frac{27}{\pi} \int_{0}^{\infty} d \omega \quad \alpha_{1}^{3}(i \omega)
$$

where we have used the definition

$$
\alpha_{1}(i \omega) \equiv \frac{2}{3} \operatorname{Re}\left[\left(n_{g} 0\left|r g_{1}\left(E_{n_{g} 0}-i \omega\right) r\right| n_{g} 0\right)\right] .
$$

Finally, the third-order correction to the ground-state energy in the lowest order of inverse powers of the internuclear distances may be written as

$$
E_{\nu_{g} \nu_{g} \nu_{g}}^{(3)}=\left(1+3 \cos \beta_{1} \cos \beta_{2} \cos \beta_{3}\right) \frac{C}{R_{12}^{3} R_{23}^{3} R_{31}^{3}},
$$

where the three-dipole interaction dispersion coefficient $C$ is given by

$$
C=\frac{3}{\pi} \int_{0}^{\infty} d \omega \quad \alpha_{1}^{3}(i \omega)
$$

This result may be easily generalized to the case of three different species of alkali-metal atoms, in which case $C$ is given by

\footnotetext{
${ }^{1} \mathrm{~A}$ similar integral representation has been used by Dalgarno and Victor [26].
}

$$
C=\frac{3}{\pi} \int_{0}^{\infty} d \omega \alpha_{1}^{(A)}(i \omega) \alpha_{1}^{(B)}(i \omega) \alpha_{1}^{(C)}(i \omega)
$$

where the superscripts $A, B$, and $C$ designate the atomic species. In this way the three-center molecular problem of computing the long-range potential surface interaction has been reduced to the one-center atomic problem of evaluating the dynamic dipole polarizabilities for each atom for imaginary frequencies. Our result in Eqs. (22) and (24) agrees with Eq. (4.3) of Ref. [8].

\section{RESULTS AND DISCUSSIONS}

\section{A. Computational procedure}

The main task in the evaluation of the three-dipole dispersion coefficients $C$, given by Eq. (23) or (24), consists primarily of calculating the atomic dipole polarizabilities $\alpha_{1}$ for imaginary frequencies using Eq. (21). They were evaluated by the Dalgarno-Lewis method [27] of solving the inhomogeneous differential equation for the linear response. Notice that the dipole operators in Eq. (21) have been replaced by their expressions from model potential theory [28-31], i.e.,

$$
r \rightarrow r\left\{1-\frac{\alpha_{c}}{r^{3}}\left[1-e^{-\left(r / r_{c}^{\prime}\right)^{3}}\right]\right\}
$$

where $\alpha_{c}$ is the core polarization of the positive ion and $r_{c}^{\prime}$ is a parameter fitted in order to reproduce the experimental values of the static dipole polarizabilities [32]. Details of the numerical methods used to compute the polarizabilities have been described in Ref. [1]. To complete the evaluation of the $C$ coefficients, one needs also to carry out the integral over $\omega$ in Eq. (23) or (24). Our codes have been tested numerically by comparing our results for the interaction of three hydrogen atoms with the result of Chan and Dalgarno [9]. We find the $C$ coefficient to be 21.64248 , which is in excellent agreement with the value 21.6425 of Ref. [9].

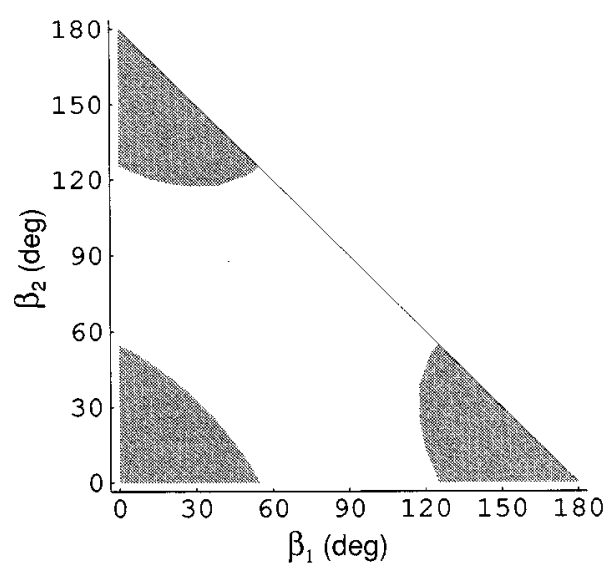

FIG. 2. Contour plot of the angular function $D\left(\beta_{1}, \beta_{2}, \beta_{3}\right)$ $=\frac{1}{9}\left(1+3 \cos \beta_{1} \cos \beta_{2} \cos \beta_{3}\right)$, where $\beta_{2}=180^{\circ}-\beta_{1}$ and $\beta_{3}=180^{\circ}$ $-\beta_{1}-\beta_{2}$. The unshaded region indicates $D \geqslant 0$ and the shaded region indicates $D<0$. 
TABLE I. Numerical values for the three-dipole dispersion coefficients $C$ for three identical alkali-metal atoms (in a.u.).

\begin{tabular}{|c|c|c|c|c|c|c|c|}
\hline \multirow[b]{2}{*}{ Atoms } & \multicolumn{7}{|c|}{$C \times 10^{-6}$} \\
\hline & Eq. $(23)^{\mathrm{a}}$ & Eq. $(27)^{b}$ & $\mathrm{SD}^{\mathrm{c}}$ & $\mathrm{LK}^{\mathrm{d}}$ & MPZM $^{\mathrm{e}}$ & $S C^{f}$ & YBDD $^{\mathrm{g}}$ \\
\hline $\mathrm{Li}$ & 0.1701 & 0.1707 & 0.169 & 0.169 & 0.1693 & 0.170 & 0.170595 \\
\hline $\mathrm{Na}$ & 0.1758 & 0.1758 & & 0.209 & & 0.176 & \\
\hline K & 0.8375 & 0.8373 & & 0.824 & & 0.861 & \\
\hline $\mathrm{Rb}$ & 1.060 & 1.060 & & 1.06 & & 1.10 & \\
\hline Cs & 1.910 & 1.910 & & 1.35 & & 1.99 & \\
\hline
\end{tabular}

${ }^{\text {apresent results. }}$

${ }^{b}$ Results obtained with approximation of Y. Midzuno and T. Kihara [5].

${ }^{c}$ Results of G.M. Stacey and A. Dalgarno [13], Table V.

${ }^{\mathrm{d}}$ Results of P.W. Langhoff and M. Karplus [16], Table XII. Result for K has an uncertainty of $\pm 0.001 \times 10^{6}$ a.u.

${ }^{\mathrm{e}}$ Results of D.J. Margoliash, T.R. Proctor, G.D. Zeiss, and W.J. Meath [15], Table 1.

${ }^{\mathrm{f}}$ Results of J.M. Standard and P.R. Certain [17], Table VII. Note that their definition of $C$ is a factor 3 smaller than ours. Result for $\mathrm{Na}$ is their lower bound; in all other cases lower and upper bounds are equal.

${ }^{g}$ Results of Z.C. Yan, J.F. Babb, A. Dalgarno, and G.W.F. Drake [14], Table X.

\section{B. Results for $C$ and its contribution to the long-range interaction potential}

In the long-range limit the potential surface interaction among three alkali-metal atoms is given by the contribution of Eq. (7) and Eq. (22), i.e.,

$$
\begin{aligned}
& V\left(\vec{R}_{12}, \vec{R}_{23}, \vec{R}_{31}\right) \\
&=-\frac{C_{6}^{(12)}}{R_{12}^{6}}-\frac{C_{8}^{(12)}}{R_{12}^{8}}-\frac{C_{10}^{(12)}}{R_{12}^{10}}-\cdots-\frac{C_{6}^{(23)}}{R_{23}^{6}}-\frac{C_{8}^{(23)}}{R_{23}^{8}}-\frac{C_{10}^{(23)}}{R_{23}^{10}} \\
&-\cdots-\frac{C_{6}^{(31)}}{R_{31}^{6}}-\frac{C_{8}^{(31)}}{R_{31}^{8}}-\frac{C_{10}^{(31)}}{R_{31}^{10}}-\cdots \\
&+\left(1+3 \cos \beta_{1} \cos \beta_{2} \cos \beta_{3}\right) \frac{C}{R_{12}^{3} R_{23}^{3} R_{31}^{3}}+\cdots
\end{aligned}
$$

Note that in Eq. (26) the three-dipole dispersion coefficient $C$ enters with a different sign from those of the pairinteraction coefficients $C_{6}, C_{8}$, and $C_{10}$. Also, $C$ is multiplied by an angular dependent factor, which is positive for $\max \left(\beta_{1}, \beta_{2}, \beta_{3}\right)<117^{\circ}$ and negative for $\max \left(\beta_{1}, \beta_{2}, \beta_{3}\right)$ $>126^{\circ}$ [5]. A contour plot showing positive and negative regions for the angular factor is given in Fig. 2.

Our numerical results for the coefficient $C$ are presented in Tables I and II. Table I presents results calculated using Eq. (23) for the case of identical alkali-metal atoms. Table II presents results calculated using Eq. (24) for all heteronuclear combinations of three alkali-metal atoms. In Table I we have compared our results obtained from Eq. (23) with results of other authors for the alkali metals [13-17]. For $\mathrm{Li}$ one sees that all results lie within the range $(0.170 \pm 0.001) \times 10^{+6}$ a.u. For the other alkali metals the only other results are the Padé approximant bounds of Refs. [16] and [17]. Although both upper and lower bounds predicted by each of those references are equal (except for $\mathrm{K}$ in the case of Ref. [16] and $\mathrm{Na}$ in the case of Ref. [17]), it is clear from Table I that the bounds predicted by these two references do not agree with each other and, with the exception of the Ref. [16] result for $\mathrm{Rb}$, do not agree with our results. We note once again the sensitivity of these bounds to the theoretical and experimental input data used $[16,17]$.

Note finally that the values of the $C$ coefficients are roughly one order of magnitude less than those of the $C_{10}$ coefficients obtained for the alkali metals in Ref. [1]. However, the contribution of the $C$ coefficients comes in the ninth order of the inverse power of the internuclear separation (whereas the $C_{10}$ coefficients contribute in the tenth order). Thus the contribution of the three-dipole interaction dispersion coefficient $C$ may be comparable to the contribution of the pair-interaction dispersion coefficient $C_{10}$, and so our results show that three-body effects may not be neglected in the description of the long-range surface potential interaction among three alkali-metal atoms.

\section{Comparison with approximate formulas for $C$}

Midzuno and Kihara [5,6] derived an approximate expression for the atomic three-dipole dispersion coefficient $C .^{2}$ For the case of three identical atoms, they obtain

$$
C=\frac{3}{4} \alpha_{1}(0) C_{6},
$$

and for the general case of three different atoms, they obtain

$$
C=\frac{2 Q_{1} Q_{2} Q_{3}\left(Q_{1}+Q_{2}+Q_{3}\right)}{\left(Q_{1}+Q_{2}\right)\left(Q_{2}+Q_{3}\right)\left(Q_{3}+Q_{1}\right)}
$$

where

\footnotetext{
${ }^{2}$ Their result is an exact one for the particular case of three interacting harmonic oscillators. McLachlan et al. [33] have shown that when two interacting atoms are treated by the Hartree method, it is equivalent to regarding each as an assembly of harmonic oscillators.
} 
TABLE II. The three-dipole interaction coefficients $C$ for all heteronuclear combinations of alkali-metal atoms in their ground states (in a.u.).

\begin{tabular}{lccccc}
\hline \hline & \multicolumn{2}{c}{$C \times 10^{-5}$} & \multicolumn{2}{c}{$C \times 10^{-5}$} \\
\multicolumn{1}{c}{ Atoms } & Eq. $(24)^{\mathrm{a}}$ & Eq. $(28)^{\mathrm{b}}$ & Atoms & Eq. $(24)^{\mathrm{a}}$ & Eq. $(28)^{\mathrm{b}}$ \\
\hline Li-Li-Na & 1.716 & 1.721 & Rb-Rb-Cs & 12.88 & 12.87 \\
Li-Li-K & 2.884 & 2.891 & Cs-Cs-Li & 8.442 & 8.448 \\
Li-Li-Rb & 3.116 & 3.124 & Cs-Cs-Na & 8.447 & 8.443 \\
Li-Li-Cs & 3.768 & 3.775 & Cs-Cs-K & 14.48 & 14.48 \\
Na-Na-Li & 1.735 & 1.737 & Cs-Cs-Rb & 15.67 & 15.67 \\
Na-Na-K & 2.928 & 2.928 & Li-Na-K & 2.904 & 2.907 \\
Na-Na-Rb & 3.161 & 3.161 & Li-Na-Rb & 3.136 & 3.140 \\
Na-Na-Cs & 3.808 & 3.806 & Li-Na-Cs & 3.785 & 3.788 \\
K-K-Li & 4.908 & 4.912 & Li-K-Rb & 5.305 & 5.311 \\
K-K-Na & 4.929 & 4.928 & Li-K-Cs & 6.429 & 6.433 \\
K-K-Rb & 9.057 & 9.056 & Li-Rb-Cs & 6.952 & 6.958 \\
K-K-Cs & 11.00 & 11.00 & Na-K-Rb & 5.327 & 5.326 \\
Rb-Rb-Li & 5.735 & 5.742 & Na-K-Cs & 6.444 & 6.442 \\
Rb-Rb-Na & 5.756 & 5.756 & Na-Rb-Cs & 6.967 & 6.965 \\
Rb-Rb-K & 9.796 & 9.796 & K-Rb-Cs & 11.90 & 11.90 \\
\hline \hline
\end{tabular}

${ }^{\text {apresent results. }}$

${ }^{b}$ Results obtained with approximation of Midzuno and Kihara [5].

$$
\frac{1}{Q_{i}}=\frac{1}{C_{6}^{(i j)} \alpha_{1}^{(k)}(0)}+\frac{1}{C_{6}^{(k i)} \alpha_{1}^{(j)}(0)}-\frac{1}{C_{6}^{(j k)} \alpha_{1}^{(i)}(0)},
$$

with $i, j, k=1,2,3$. Chan and Dalgarno [9] found that using Eq. (27) for three interacting hydrogen atoms gave a result that was only $1.2 \%$ larger than their result for $C$. Similar comparisons in Ref. [10] for three identical noble gas atoms gave results within 5\% of those of direct calculations. Given this close agreement, we have therefore also compared the results of using the approximate Eqs. (27) and (28) with our results obtained from Eqs. (23) and (24), respectively. The two sets of results are compared in Tables I and II. We see that in all cases, the approximate Eqs. (27) and (28) give agreement with the results of Eqs. (23) and (24) to better than $0.4 \%$, where the largest discrepancy is for the case of three $\mathrm{Li}$ atoms.

The approximate formulas of Midzuno and Kihara [5,6] led Tang [18] to develop two similar approximate formulas for the three-body dispersion coefficients. These formulas as well as those of Refs. [5,6] have been discussed by Margoliash et al. [15] and by Jhanwar and Meath [20]. In addition to comparing our results obtained from Eqs. (23) and (24) with those predicted by the approximate Eqs. (27) and (28), respectively, of Midzuno and Kihara [5], we have also compared our results with the approximate formulas of Tang [18]. We have not presented our latter results in Tables I and II for reasons of space limitations and because the results may be stated succinctly. Tang's first combination rule [18] assumes knowledge of the dipole-dipole dispersion coefficients for two identical atoms as well as the dipole polarizabilities of each atom involved. Note that in our calculations we have actually used the formulas of Tang given in Ref. [15]: Tang's first combination rule is obtained by combining
Eqs. (4) and (6) of Ref. [15]. The formula is given by Eq. (28) with

$$
Q_{i}=\frac{\alpha_{1}^{(j)}(0) \alpha_{1}^{(k)}(0)}{\alpha_{1}^{(i)}(0)} C_{6}^{(i i)}
$$

The results using this formula agree with those obtained from the approximate formulas of Midzuno and Kihara [5] that we present in Tables I and II to within $\pm 0.0002 \times 10^{+6}$ a.u. in all cases. Tang's second combination rule [which we obtain by combining Eqs. (4) and (8) of Ref. [15]] assumes knowledge of the three-body dispersion coefficient $C^{(i i i)}$ for three identical atoms as well as the dipole polarizabilities of each atom. It is given by Eq. (28) with

$$
Q_{i}=\frac{4}{3} \frac{\alpha_{1}^{(j)}(0) \alpha_{1}^{(k)}(0)}{\left[\alpha_{1}^{(i)}(0)\right]^{2}} C^{(i i i)}
$$

This second combination rule permits one to obtain the three-body dispersion coefficients for nonidentical combinations of three interacting atoms. The results (when we use our predictions for the three-body dispersion coefficients for three identical atoms from Table I) agree with our predictions in Table II for nonidentical combinations of three interacting atoms to within $\pm 0.002 \times 10^{+5}$ a.u. in all cases.

\section{Dependence of $\boldsymbol{C}$ on the static dipole polarizabilities}

Since the numerical values of the $C$ coefficients depend strongly on the accuracy of the atomic dipole polarizabilities, one may ask what is the effect of the uncertainty in the experimental value of the static dipole polarizability on the evaluation of the $C$ coefficients. In our computation the 
TABLE III. Dependence of the three-dipole dispersion coefficient $C$ for three $\mathrm{Na}$ atoms on selected values of the static dipole polarizability $\alpha_{1}(0)$. Model potential cutoff radii, $r_{c}^{\prime}$, corresponding to each value of $\alpha_{1}(0)$, are also shown.

\begin{tabular}{ccc}
\hline \hline$\alpha_{1}(0)$ (a.u.) & $r_{c}^{\prime}$ (a.u.) & $C\left(10^{5}\right.$ a.u. $)$ \\
\hline 158.8 & 0.2789111 & 1.744 \\
$159.2^{\text {a }}$ & 0.3798826 & 1.758 \\
160.1 & 0.5692351 & 1.788 \\
161.0 & 0.9396196 & 1.819 \\
161.9 & 2.0587556 & 1.850 \\
$162.7^{\text {b }}$ & 2.8608128 & 1.877 \\
163.5 & 3.7176877 & 1.905 \\
$164.7^{\text {c }}$ & 5.7251209 & 1.948 \\
165.5 & 9.9588996 & 1.976 \\
\hline \hline
\end{tabular}

${ }^{\mathrm{a}}$ Experimental result of Ref. [32].

${ }^{b}$ Experimental result of Ref. [35].

${ }^{c}$ Experimental result of Ref. [34].

value of the static dipole polarizabilities has been fixed to the widely-used experimental values of Molof et al. [32] by adjusting the $r_{c}^{\prime}$ parameter [1] in the dipole operator expression in Eq. (25). Then the dynamic dipole polarizabilities for imaginary frequencies have been evaluated using Eq. (21) and the $C$ coefficients have been subsequently evaluated from Eqs. (23) and (24). In order to determine the effect of experimental static dipole polarizability uncertainties on our calculated $C$ coefficients we have repeated our calculations for various static dipole polarizability values. To illustrate our findings, consider the case of three $\mathrm{Na}$ atoms. In Table III we present our results for the $C$ coefficient for selected values of $\alpha_{1}(0)$, together with the corresponding model potential parameters $r_{c}^{\prime}$. Among the $\mathrm{Na} \alpha_{1}(0)$ values included, we selected the experimental results of Molof et al. [32], of Hall and Zorn [34], and of Ekstrom et al. [35]. In Fig. 3 we present a plot of the $C$ coefficient versus $\alpha_{1}(0)$. The three experimental values included in Table III are indicated in Fig. 3 by black circles. Other values of $\alpha_{1}(0)$ used to compute $C$ are indicated in Fig. 3 by open circles. Figure 3 shows that the $C$ coefficient has an almost linear dependence on small variations in the value of $\alpha_{1}(0)$. Analyzing the numerical values obtained, we conclude that the relative uncertainty in the $C$ coefficient is roughly three times bigger than the relative uncertainty in the static dipole polarizability $\alpha_{1}(0)$. This result may be understood as follows: in Eq. (23) the dipole polarizability enters with a power of 3 and the main contribution to the integral in Eq. (23) is given by small values of $\omega$ (i.e., the static dipole polarizability gives the dominant contribution). This result is expected to hold for the $C$ coefficients for any three interacting atoms. Thus in a similar way, we expect that the numerical values for the $C$ coefficients presented in Table II for all heteronuclear combinations of three alkali-metal atoms will have uncertainties given by the sum of the relative uncertainties in the static dipole polarizabilities, as may be inferred from Eq. (24) and the fact that the main contribution to the integral is for small values of $\omega$. Also we mention that Table III may be used to

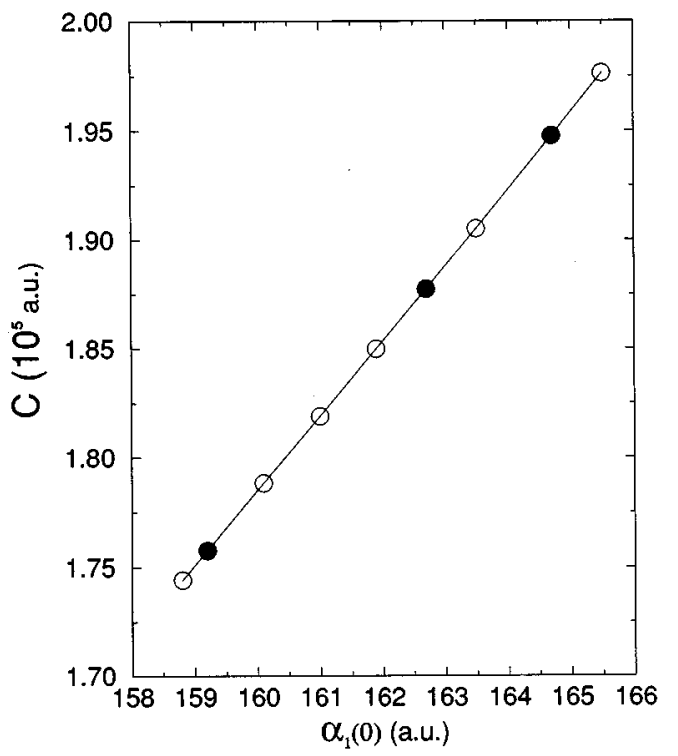

FIG. 3. Dependence of the three-dipole dispersion coefficient $C$ on the static dipole polarizability $\alpha_{1}(0)$ for the case of three $\mathrm{Na}$ atoms. The results for $C$ corresponding to the experimental values of the static dipole polarizability given in Refs. [32], [35], and [34] are indicated by the three black circles in the order from left to right, respectively.

estimate by interpolation the $C$ coefficient for three Na atoms for any other static dipole polarizability value.

\section{CONCLUSIONS}

Using nondegenerate perturbation theory up to third order, we have computed the dipole dispersion coefficient $C$ for three interacting alkali-metal atoms. The $C$ coefficient measures the strength of the nonadditive part of the longrange three-atom interaction. Numerical computations have been carried out for all possible combinations of three alkalimetal atoms. The values obtained indicate that such threebody nonadditive dipole interaction effects are comparable in magnitude to those of the two-body dipole-octupole and quadrupole-quadrupole interaction effects. Thus they may not be neglected in the description of the long-range surface potential interaction for alkali-metal atoms.

Our results for three-body dispersion coefficients have been compared with results of other authors for the case of three identical interacting atoms. While there is theoretical agreement in the case of $\mathrm{Li}$, prior estimates for the other alkali metals in general differ significantly from our present results. In all cases we have compared our present results for $C$ with those resulting from the approximate formulas of Midzuno and Kihara [5,6]. There is excellent agreement for the case of alkali-metal atoms. We have also evaluated approximate formulas of Tang [18] and find in all cases there is nearly identical agreement with either our results or with results of the approximate formulas of Midzuno and Kihara $[5,6]$, depending on the formula used. Thus we conclude that the approximate formulas (or combination rules) give reliable results when accurate input data are used.

Finally, based on a numerical analysis carried out for the case of three $\mathrm{Na}$ atoms, we have concluded that, in general, the relative uncertainty in each of our results for the $C$ coef- 
ficient is roughly three times bigger than the relative uncertainty in the experimentally measured value of the static dipole polarizability $\alpha_{1}(0)$ for the case of three identical interacting atoms and is roughly equal to the sum of the relative uncertainties in the experimentally measured static dipole polarizabilities for the case of three different interacting atoms.

\section{ACKNOWLEDGMENTS}

We thank Alexander Dalgarno for fruitful discussions concerning the three-body dispersion forces. This work was supported in part by the U.S. Department of Energy, Division of Chemical Sciences, Office of Basic Energy Sciences, under Grant No. DE-FG02-88ER13955.
[1] M. Marinescu, H.R. Sadeghpour, and A. Dalgarno, Phys. Rev. A 49, 982 (1994).

[2] B.M. Axilrod and E. Teller, J. Chem. Phys. 11, 299 (1943).

[3] Y. Muto, Nippon Suugaku-Butsuri Gakkaisi 17, 629 (1943).

[4] B.M. Axilrod, J. Chem. Phys. 19, 719 (1951).

[5] Y. Midzuno and T. Kihara, J. Phys. Soc. Jpn 11, 1045 (1956).

[6] T. Kihara, Adv. Chem. Phys. 1, 267 (1958).

[7] M.R. Aub and S. Zienau, Proc. R. Soc. London Ser. A 257, 464 (1960).

[8] A.D. McLachlan, Mol. Phys. 6, 423 (1963).

[9] Y.M. Chan and A. Dalgarno, Mol. Phys. 9, 525 (1965).

[10] A. Dalgarno and W.D. Davison, Adv. Atom. Mol. Phys. 2, 1 (1965).

[11] H.W. Graben and R.D. Present, Phys. Rev. Lett. 9, 247 (1962).

[12] A.E. Sherwood and J.M. Prausnitz, J. Chem. Phys. 41, 413 (1964); 41, 429 (1964).

[13] G.M. Stacey and A. Dalgarno, J. Chem. Phys. 48, 2515 (1968).

[14] Z.-C. Yan, J.F. Babb, A. Dalgarno, and G.W.F. Drake, Phys. Rev. A 54, 2824 (1996).

[15] D.J. Margoliash, T.R. Proctor, G.D. Zeiss, and W.J. Meath, Mol. Phys. 35, 747 (1978).

[16] P.W. Langhoff and M. Karplus, J. Chem. Phys. 53, 233 (1970).

[17] J.M. Standard and P.R. Certain, J. Chem. Phys. 83, 3002 (1985).

[18] K.T. Tang, Phys. Rev. 177, 108 (1969).

[19] H.L. Kramer and D.R. Herschbach, J. Chem. Phys. 53, 2792 (1970).
[20] B.L. Jhanwar and W.J. Meath, Mol. Phys. 41, 1061 (1980).

[21] B.C. Carlson and G.S. Rushbrooke, Proc. Cambridge Philos. Soc. 46, 626 (1950).

[22] R.J. Buehler and J.O. Hirschfelder, Phys. Rev. 83, 628 (1951).

[23] M.E. Rose, J. Math. Phys. 37, 215 (1958).

[24] M. Marinescu and A. Dalgarno, Phys. Rev. A 52, 311 (1995).

[25] D.A. Varshalovich, A.N. Moskalev, and V.K. Khersonskii, Quantum Theory of Angular Momentum (World Scientific, Singapore, 1988), p. 119.

[26] A. Dalgarno and G.A. Victor, Mol. Phys. 10, 333 (1966), Eq. (15).

[27] A. Dalgarno and J.T. Lewis, Proc. R. Soc. London Ser. A 233, 70 (1955).

[28] I.B. Bersuker, Opt. Spektrosk. 3, 97 (1957).

[29] S. Hameed, A. Herzenberg, and M.G. James, J. Phys. B 1, 822 (1968).

[30] T.C. Caves and A. Dalgarno, J. Quant. Spectrosc. Radiat. Trans. 12, 1539 (1972).

[31] C. Bottcher and A. Dalgarno, Proc R. Soc. London Ser. A 340, 187 (1974).

[32] R.W. Molof, H.J. Schwartz, T.M. Miller, and B. Bederson, Phys. Rev. A 10, 1131 (1974).

[33] A.D. McLachlan, R.D. Gregory, and M.A. Ball, Mol. Phys. 7, 119 (1963).

[34] W.D. Hall and J.C. Zorn, Phys. Rev. A 10, 1141 (1974).

[35] C.R. Ekstrom, J. Schmiedmayer, M.S. Chapman, T.D. Hammond, and D.E. Pritchard, Phys. Rev. A 51, 3883 (1995). 